\title{
Hipotiroidismo subclínico en mujeres en edad reproductiva y embarazadas
}

\author{
Subclinical hypothyroidism in women of reproductive age and in pregnancy
}

Ivan Arauco $^{\mathrm{a}}$, Nadia Sgarbossa ${ }^{\mathrm{a}}$, Juan Victor Ariel Franco ${ }^{\mathrm{b}, \mathrm{c}, \mathrm{d}}$

\begin{abstract}
Resumen
El hipotiroidismo subclínico suele ser identificado como un cuadro que disminuye la capacidad reproductiva de las mujeres y está asociado a un riesgo aumentado de complicaciones perinatales. A partir de un caso clínico real, revisamos la evidencia disponible y encontramos que existen pruebas que contradicen este conocimiento tradicional sobre el pronóstico y la necesidad de tratamiento de este cuadro.
\end{abstract}

\begin{abstract}
Subclinical hypothyroidism is usually identified as a condition that decreases the reproductive capacity of women and is associated with a higher risk of perinatal complications. From a real clinical case, we review the available evidence and found that there is evidence that contradicts this traditional knowledge about the prognosis and the need for treatment of this condition.
\end{abstract}

Palabras clave: Hipotiroidismo, Embarazo, Reproducción, Fertilidad. Keywords: Hypothyroidism, Pregnancy, Reproduction, Fertility.

Arauco I, Sgarbossa N, Franco JVA. Hipotiroidismo subclínico en mujeres en edad reproductiva y embarazadas. Evid Actual Pract Ambul. 2019;22(2):e002015.

\section{Introducción}

\section{Escenario clínico}

Una mujer de 28 años consulta con su médico de cabecera para hacerse un control de salud y refiere que hace un año está intentando quedar embarazada, pero no puede. Concurre acompañada de su pareja, un hombre de 30 años de edad. Ambos conviven desde hace 5 años en un departamento, en una ciudad del conurbano bonaerense. Ella menciona que últimamente se encuentra muy ansiosa porque quiere formar una familia, pero teme que sea infértil. Refiere tener ciclos menstruales regulares; su fecha de última menstruación fue en el último mes. Es sana y tiene antecedentes familiares (madre y abuela) de hipotiroidismo. En sus análisis de laboratorio tiene hormonas T3 y T4 normales, pero la TSH ligeramente elevada $(7,10 \mathrm{mUI} / \mathrm{L})$.

\section{Pregunta que generó el caso}

¿Cuál es el pronóstico del hipotiroidismo subclínico en relación con la capacidad y desenlaces reproductivos en mujeres de edad fértil? (pregunta pronóstica) ¿El tratamiento con T4, lo cambia? (pregunta terapéutica)

\section{Estrategia de búsqueda}

Se realizó una búsqueda de datos en PubMed y Cochrane Library en junio de 2019. Los términos utilizados en distintas combinaciones fueron subclinicalhypothyroidism, pregnancy, y fertility. Se seleccionaron 3 artículos que parecían ser los más relevantes para responder a las preguntas planteadas.

\section{Algunas consideraciones sobre el hipotiroidismo sub- clínico}

Con el término hipotiroidismo primario se hace referencia al déficit hormonal causado por fallo intrínseco de la glándula tiroides, que interrumpe la síntesis y la secreción de T4 y T3. El hipotiroidismo subclínico (HSC) o leve es un tipo de hipotiroidismo asintomático o con presencia de síntomas leves, en el cual el nivel de TSH presenta un aumento moderado, mientras que el nivel de T4 libre plasmático sigue en el intervalo normal bajo a normal. E hipotiroidismo primario está presente en casi el $5 \%$ de los individuos. Se diagnostica con mayor frecuencia en mujeres y a medida que avanza la edad. La deficiencia de yodo en la dieta es la principal causa de hipotiroidismo primario en determinadas regiones subdesarrolladas del mundo, mientras que la causa más frecuente en la mayoría de los países es la tiroiditis autoinmunitaria o de Hashimoto ${ }^{1}$. Los síntomas de hipotiroidismo incluyen fatiga, letargia, aumento de peso a pesar de la pérdida del apetito intolerancia al frío, ronquera, estreñimiento, debilidad, mialgias, artralgias, parestesias, sequedad de la piel y caída del pelo. Las mujeres pueden desarrollar pubertad precoz, menorragia, amenorrea y galactorrea. Las personas afectadas pueden desarrollar depresión, con iniciativa y sociabilidad limitadas. Las deficiencias cognitivas pueden oscilar entre lapsus leves de la memoria y el delirio, la demencia, las convulsiones y el coma. Sin embargo en el HSC, los síntomas suelen no estar presentes o ser muy leves. Los intervalos de referencia establecidos para los niveles de TSH se encuentran normalmente entre 0,5 y $4,5 \mathrm{mUI}^{2}$.

\section{Resumen de la evidencia}

Para la realización de este trabajo, se analizaron los resultados de 3 investigaciones:

\section{Plowden TC, et al. Subclinical Hypothyroidism and Thyroid Autoimmunity Are Not Associated With Fecundity, Pregnancy Loss, or Live Birth. J Clin Endocrinol Metab. 2016 Jun;101(6):2358-65}

Este estudio de cohorte prospectivo ${ }^{3}$, realizado en 4 centros médicos de EE.UU. entre 2007 y 2010, apoyado por el Programa de Investigación Intramural del Instituto Nacional de Salud Infantil y Desarrollo Humano Eunice Kennedy Shriver, deriva de un ensayo clínico que había estudiado los efectos de la aspirina a bajas dosis en la gestión y la reproducción ${ }^{4}$. Las participantes fueron 1.228 mujeres, de 18 a 40 años, que intentaban concebir y

\footnotetext{
a Departamento de Salud, Universidad Nacional de La Matanza (Carrera de Medicina). ivan.arauco@outlook.com nadia.sgarbossa1@gmail.com

b Departamento de Investigación, Instituto Universitario Hospital Italiano.

c Servicio de Medicina Familiar y Comunitaria, Hospital Italiano de Buenos Aires.

d Departamento de Salud, Universidad Nacional de La Matanza. juan.franco@hospitalitaliano.org.ar
} 
tenían uno o dos embarazos perdidos previos, sin antecedentes de infertilidad (primaria). De manera simultánea se buscó examinar las asociaciones entre las concentraciones de TSH antes del embarazo y la autoinmunidad tiroidea con el tiempo hasta el embarazo, la pérdida del embarazo y el parto vivo. Un total de 566 mujeres con TSH sérica de menos de $2.5 \mathrm{mUI} / \mathrm{L}$ quedaron embarazadas $(566 / 884,64,0 \%)$ vs $205(205 / 303,67,7 \%)$ con al menos $2,5 \mathrm{mUl} / \mathrm{L}(p=0,25)$. Las mujeres con TSH mayor o igual a 2,5 $\mathrm{mIU} / \mathrm{L}$ no tuvieron mayor riesgo de pérdida de embarazo (Riesgo Relativo [RR] 1,07; intervalo de confianza del 95\% [IC 95\%], 0,81 a 1,41) o disminución en la tasa de nacidos vivos (RR, 0,97; IC $95 \%$ IC, 0,88 a 1,07) o del tiempo al embarazo (odds ratio de fecundidad, 1,09; IC $95 \%, 0,90$ a 1,31) en comparación con las mujeres con TSH menor de $2,5 \mathrm{mIU} / \mathrm{L}$, después del ajuste por edad e índice de masa corporal.

Este estudio tiene fortalezas metodológicas con respecto a otros realizados con anterioridad, como la inclusión y determinación de niveles hormonales de las participantes antes de la concepción, y la utilización de modelos lineales generalizados ajustados por la edad e IMC. Sus resultados sustentan la recomendación del Congreso Americano de Obstetras y Ginecólogos, quienes no recomiendan el examen de TSH en pacientes sanas, de bajo riesgo, asintomáticas. Como debilidad podemos nombrar que el seguimiento de las pacientes se suspendió si no quedaban embarazadas después de seis ciclos o después de experimentar una segunda pérdida de periconcepción durante el estudio, y dado que no se especificó el número de éstas exclusiones, no se puede descartar un posible subregistro en los resultados obtenidos.

\section{Reid SM, et al. Interventions for clinical and subclinical} hypothyroidism pre-pregnancy and during pregnancy. Cochrane Database Syst Rev. 2013 May 31;(5):CD007752

Para la realización de esta revisión sistemática ${ }^{5}$, se realizó una búsqueda en el registro de ensayos del grupo Cochrane Pregnancy and Childbirth Group's Trials Register (hasta marzo de 2013) y se incluyeron ensayos controlados aleatorizados (ECA) y ensayos controlados cuasialeatorizados que compararan una intervención farmacológica para el hipotiroidismo primario o el HSC, antes o durante el embarazo, con otra intervención o placebo.

En uno de los ensayos, 115 embarazadas eutiroideas con anticuerpos antiperoxidasas fueron divididas en dos grupos; uno fue tratado con levotiroxina mientras el otro no. No se redujo la incidencia de preeclampsia (RR 0,61; IC 95\%: 0,11 a 3,48), pero sí se redujo significativamente la incidencia de parto prematuro en $72 \%$ (RR 0,28; IC $95 \%$ 0,10 a 0,80).

En dos de los ensayos, con 30 y 48 mujeres hipotiroideas respectivamente, se compararon diferentes dosis de levotiroxina, pero ambos estudios informaron solo resultados bioquímicos. En el cuarto ensayo, 169 mujeres fueron tratadas con 20 ug/día de selenio, mientras que el grupo control recibió placebo; no se observaron diferencias para la incidencia de preeclampsia (RR 1,44; IC $95 \%$ : 0,25 a 8,38) o parto prematuro (RR 0,96; IC $95 \%$ : 0,20 a 4,61). El riesgo de sesgo en los estudios fue incierto 0 alto en la mayoría de los casos.

La debilidad principal fue la muestra reducida en cada uno de los cuatro estudios incluidos, con resultados neutros para la mayoría de los desenlaces, y el énfasis en resultados bioquímicos (desenlaces subrogados).

\section{Akhtar MA, et al. Thyroxine replacement for subfertile women with euthyroid autoimmune thyroid disease or subclinical hypothyroidism. Cochrane Database Syst Rev. 2019 Jun 25;6:CD011009}

Para realizar esta revisión sistemática ${ }^{6}$, se buscaron ECA (hasta abril de 2019), en el registro especializado del Grupo Cochrane de Ginecología y Fertilidad, CENTRAL, MEDLINE, Embase, PsycINFO, CINAHL y dos registros de ensayos clínicos. Adicionalmente, los revisores verificaron las referencias y se contactaron con autores de estudios y expertos en el campo para identificar estudios potencialmente relevantes.

Se incluyeron cuatro ECA con 820 mujeres en total. Las participantes eran mujeres en tratamiento de reproducción asistida, tanto fertilización in vitro (FIV) como inyección intracitoplasmática de esperma (ICSI), con antecedentes de subfertilidad y con HSC o con enfermedad tiroidea autoinmune eutiroidea (ATAE). Se excluyeron las mujeres con hipotiroidismo clínico previamente conocido o que ya se encontraran medicadas con tiroxina.

Un estudio con 64 mujeres con HSC, encontró evidencia de baja calidad que sugiere que el reemplazo de levotiroxina puede haber mejorado la tasa de nacimientos vivos (RR 2,13; IC $95 \%$ : 1,07 a 4,21) y puede haber llevado a tasas de aborto espontáneo similares (RR 0,11; IC $95 \%$ : 0,01 a 1,98)

En mujeres con ATAE, baja calidad de evidencia proveniente de dos ensayos clínicos con 686 mujeres sugiere que el tratamiento con reemplazo de levotiroxina en comparación con placebo o ningún tratamiento puede haber llevado a tasas similares de nacidos vivos (RR 1,04, IC $95 \% 0,83$ a 1,29) y de aborto espontáneo (RR 0,83; IC $95 \%$ : 0,47 a 1,46).

Ninguno de los ECA incluidos informó sobre efectos adversos directos de la tiroxina o complicaciones en el embarazo -maternos o fetales- que estuviesen relacionados a este tratamiento.

La calidad de la evidencia fue baja por problemas metodológicos e imprecisión, por lo que se puede aseverar que existe incertidumbre sobre si la suplementación hormonal con levotiroxina mejoraría los resultados de fertilidad después de la FIV o ICSI para mujeres diagnosticadas con HSC o ATAE.

\section{Conclusiones}

A partir de la información que hemos recabado, podemos decir que el pronóstico de las mujeres con HSC con respecto a su capacidad reproductiva es bueno, ya que, si bien se ha documentado una disminución en el número de nacidos vivos, la diferencia con respecto a las mujeres eutiroideas no es significativa. La evidencia disponible es insuficiente para aseverar que las mujeres con HSC no necesitarían ser tratadas para evitar complicaciones sobre su capacidad reproductiva y perinatales. Por otro lado, la prescripción de levotiroxina a toda mujer con HSC que busca ser madre podría exponerla a los diversos efectos adversos de este fármaco, suponiendo una disminución en su calidad de vida en pos de un beneficio que no está totalmente demostrado. Debido a esta incertidumbre en el balance de beneficios y riesgos, concluimos que la decisión de administrar levotiroxina a las mujeres hipotiroideas debería ser compartida con las pacientes, jerarquizando sus valores y preferencias individuales, ya que es necesario tener en cuenta que el deseo de ser madre podría ser más importante para la paciente que las posibles complicaciones y costos que podría suponerle el tratamiento.

\section{Referencias}

1. Cap. 230 Enfermedades de la glándula tiroides. In: Rozman C, Cardellach F, editors. Farreras Rozman. Medicina Interna. España: Elsevier; 2016.

2. Goldman L, Schafer A. Cap. 226 Tiroides. In: Goldman-Cecil: Tratado de medicina interna. España: Elsevier Castellano; 2017.

3. Plowden TC, Schisterman EF, Sjaarda LA, Zarek SM, Perkins NJ, Silver R, et al. Subclinical Hypothyroidism and Thyroid Autoimmunity Are Not Associated With Fecundity, Pregnancy Loss, or Live Birth. The Journal of clinical endocrinology and metabolism. 2016 jun;101(6):2358-65. Available 
from: $10.1210 / j c .2016-1049$

4. Schisterman EF, Silver RM, Perkins NJ, Mumford SL, Whitcomb BW, Stanford JB, et al. A randomised trial to evaluate the effects of low-dose aspirin in gestation and reproduction: Design and baseline characteristics. Paediatr Perinat Epidemiol. 2013;27(6):598-609. Available from: 10.1111/ppe. 12088.

5. Reid SM, Middleton P, Cossich MC, Crowther CA, Bain E. Interventions for clinical and subclinical hypothyroidism pre-pregnancy and during pregnancy. The Cochrane database of systematic reviews. 2013 may;(5):007752. Available from: 10.1002/14651858.CD007752.pub3.

6. Akhtar MA, Agrawal R, Brown J, Sajjad Y, Craciunas L. Thyroxine replacement for subfertile women with euthyroid autoimmune thyroid disease or subclinical hypothyroidism. The Cochrane database of systematic reviews. 2019;6:011009. Available from: 10.1002/14651858.CD011009.pub2. 